\title{
Chemical modelling of multicomponent mixtures: quality assurance is more than just equilibrium data quality assessment
}

\author{
Peter M. May $\cdot$ Montserrat Filella
}

Received: 22 April 2010/Accepted: 18 August 2010/Published online: 3 September 2010

(C) Springer-Verlag 2010

\begin{abstract}
Despite the large amount of data available, the great effort put into searches for the 'best' parameters and many comparative modelling studies, considerable uncertainties continue to plague chemical thermodynamics. An important factor in this ongoing failure has been the notion that the problem can be solved by better assessment of data quality on a case-by-case basis. This approach has proved strikingly unsuccessful. A different methodology must therefore be found to meet the general requirements of thermodynamic modelling in aquatic chemistry. This paper discusses current practices in quality assessment of thermodynamic data and the problems associated with them. It outlines a general approach which might address the above problem based on two concepts: (i) using large databases to store as much of the available data as possible in the form that it appears in the literature along with an assessed 'score' or 'measure of information content' and (ii) then using automatic mechanisms informed by this score to produce the thermodynamically consistent datasets needed for modelling calculations.
\end{abstract}

This article is part of the Topical Issue "Quality Assurance of Thermodynamic Data".

P. M. May

School of Chemical and Mathematical Sciences,

Murdoch University, Murdoch, WA 6150, Australia

M. Filella ( $\bowtie)$

Institute F.-A. Forel, University of Geneva, 10 route de Suisse,

1290 Versoix, Switzerland

e-mail: montserrat.filella@unige.ch

M. Filella

SCHEMA, 92 rue Principale,

6990 Rameldange, Luxembourg
Keywords Equilibrium constants .

Speciation modelling · Multicomponent mixtures .

Quality assurance · Aquatic chemistry

\section{Introduction}

Einstein considered thermodynamics was the only physical theory that was so well based on experiment that it would never be overthrown (quoted by Brecher in [1]). It is therefore ironic that, despite the large amount of data available, great uncertainties still prevail regarding chemical thermodynamics.

The thermodynamics of aqueous solution chemistry is especially difficult. First, many systems exhibit large deviations from ideal behaviour even at quite low concentrations, because they are dominated by electrically charged species. Since as yet there is no good fundamental theory to describe the thermodynamic behaviour of concentrated aqueous electrolyte solutions, it has been necessary to use empirical approaches, with all the limitations that this implies (as described further below). Second, a very serious challenge is posed by the rich diversity of chemical reactions taking place in water, which has made it impractical to treat them all in a way that is truly comprehensive and systematic. Third, there is a poorly recognised but general loss of chemical information from the initial experimentation stage, through the processing of the experimental data and then to the description ultimately recorded in the chemical literature. All these defects bear adversely on the quality of the thermodynamic parameters available and of the calculations (models) which use them.

For these reasons, serious discrepancies appear between published equilibrium constants, enthalpies of reaction, 
heat capacities, etc., and these, in turn, have impacted badly in comparative modelling studies held between research groups for the purpose of determining best practice (in respect of both experimental technique and methods of data manipulation).

While these disturbing outcomes are known well enough, and have been for a long time, the problem itself has evidently remained intractable. An important factor in this ongoing failure has been the notion that the problem can be solved simply by better assessment of data quality on a case-by-case basis. Thus, highly reputable groups of researchers such as those engaged in compiling the Nuclear Energy Agency (NEA), Organisation for Economic Cooperation and Development (OECD), volumes on 'Chemical Thermodynamics' have pursued relentlessly original data sources and have performed on these assembled data a rigorous critical assessment by the world's leading experts. These laborious efforts have undoubtedly led to much improved thermodynamic parameters for the chemical systems which have been evaluated. However, there is no sign from all this work that the overarching problem of chemical modelling uncertainty has been solved. This is because (i) the more data that are rejected, the smaller the scope of systems which can be satisfactorily modelled and (ii) there will always remain outstanding a very large number of chemical systems-especially mixtures-which have not, and cannot possibly be, subjected to the same resource-intensive, narrowly focused assessment procedure. In other words, the need for thermodynamic data to model multicomponent electrolyte systems such as seawater is so vast that quality assurance by conventional means is simply prohibitive. The open-ended nature of thermodynamic data critical assessment is well illustrated by the periodic substantive revisions of even the most authoritative of databanks, including quite recently that of the NEA (see [2]).

It follows that a different approach must be found to meet the general requirements of thermodynamic modelling in aquatic chemistry. This paper outlines a general attack on the above problem which hinges on two concepts: (i) storing as much of the available data as possible in large databases along with an assessed 'score' or 'measure of information content' and (ii) using this assigned information content to inform automatic mechanisms that then produce the thermodynamically consistent dataset which is used for model calculations. These databases should be designed to hold the available data generally so that values appear in a way that matches the literature source as closely as possible. The 'information content' ascribed initially to the thermodynamic values reported in the literature needs to be carried through all these data manipulations to determine the final parameters used for modelling. In essence, this procedure allows the selection of data that maximises the overall thermodynamic information available within the constraints imposed by thermodynamic consistency. The hope is that this will lead progressively to modelling calculations that are more robust than they have been previously. It should also provide greater transparency, clearly linking modelling outcomes to the source of the determining data. The thermodynamic parameters of choice will be, of course, still subject to revision-sometimes dramatically-but the changes should be less chaotic than previously because of the inertia which can be expected to grow as the thermodynamic databases become increasingly reliable, i.e. well informed. The key idea is to get away from a black-andwhite perspective of values reported in the literature and, rather, to recognise that all experimental data and the thermodynamic parameters derived from them have a varying degree of information content, which can be assessed and, hence, exploited.

\section{Current practice: quality assessment of individual studies and choice of 'best' constants}

As stated in the introduction, the currently accepted approach is based on the assumption that uncertainty in chemical thermodynamic calculations can be addressed simply by a more rigorous assessment of data quality on a case-by-case basis. This methodology always leads to a singular selected 'best' set of thermodynamic parameters, as briefly described below.

Although, in principle, the only way to assure accuracy of thermodynamic data is to seek agreement between experimental values from independent researchers, this is not practical, given the number of possible chemical reactions which need to be assessed. For instance, the JESS database now has thermodynamic parameters associated with more than 75000 chemical reactions [3]. Moreover, many thousands of chemical reactions of potential interest have not yet been adequately characterised. For this reason, researchers have tried to extract the best possible information from published data by evaluating them. The caveats generated by these assessment exercises, in particular in the case of the equilibrium constants, have been the subject of many recommendations (e.g., [4-7]). These guidelines are now relatively well established and need not be discussed here in detail. Briefly, when judging the quality of the data, it is well known that one should look for (i) adequate underlying theory, (ii) correct experimental technique, (iii) correct data treatment and (iv) data reporting adequacy. These are the principles of assessment used to choose the 'best' set of constants when compiling so-called critically evaluated datasets (e.g., NIST [8] and NEA [9] critical compilations, IUPAC Critical Surveys of Stability Constants [10-13]) as 
well as databases in computer speciation codes (e.g., MINTEQA2 [14], PHREEQC [15].). Unfortunately, such guidelines provide at best the necessary set of conditions for the data to be useful. In other words, they help to eliminate entirely unsuitable values. However, they are insufficient, particularly because they cannot effectively evaluate the likely accuracy of the data. Real assessment requires mechanisms which are difficult to codify because they are subjective (e.g., assuming that data coming from reputable laboratories are more likely than average to be of good quality). The importance of such mechanisms in the final selection of data has been acknowledged by well-known critical compilers [16].

Chemical speciation calculations are typically performed by using computer modelling packages that are coupled with their own thermodynamic databases. Such databases have usually been built by applying the criteria mentioned above. However, they generally suffer from certain additional problems, namely (i) the origin in the chemical literature of the stored thermodynamic parameters is generally obscure; (ii) the record of why particular literature values have been selected in preference to others is generally inadequate; (iii) sufficient documentation of why certain data have been rejected are rarely, if ever, available; (iv) gross errors can persist in such database for many years before they are detected [17, 18] and (v) standard state values tend to be stored without the corresponding extrapolation functions and parameters used in their derivation. All these problems are a direct consequence of the manner in which thermodynamic data have traditionally been handled. While problems (i)-(iii) are, in principle, relatively easy to remedy, points (iv) and (v) are not; these therefore merit some further discussion.

\section{A recommended change of approach}

First step: building databases which store assessed information content

Current practices are characterised by a significant loss of information content. This loss occurs throughout the process of selecting the 'best' set of thermodynamic parameters and is inherent in any selection procedure based on a choice of the 'best' set of constants (i.e. involves disregarding other measured values). The process also suffers from a high likelihood of errors arising from data transcription and manipulation. Both problems can be significantly counteracted by retaining (in appropriately designed databases) all available data, along with a corresponding 'score' or 'measure of information content' to reflect the assessed quality of each datum. The chemical judgement and opinion of the experts who assign weights to the different data according to its perceived quality is therefore still required. However, information is then not being discarded, and the inherently subjective nature of the data quality assessment is made explicit.

The origin of many problems with existing databases is the fact that their data are represented in a form different from their source in the literature. Common errors include (i) recording the wrong sign of the logarithm of equilibrium constants - a surprisingly frequent error even in well-known critical compilations-and (ii) mistaken manipulations needed to transform the chemical reactions as expressed in the literature source into the form used by the database, particularly regarding the predetermined choice of species whose concentrations or activities are to be treated as unknown (the so-called master or basis species). The only way of minimising these errors effectively is to represent data in databases in a form as close as possible to the original representation. This includes storing the conditions under which the data were determined, without manipulation. For instance, the widespread practice of storing equilibrium constants in modelling package databases only for standard state conditions (i.e. at infinite dilution of solutions) should be avoided. This practice requires the use of theoretical models and associated parameters that are generally obscure to the user with the result that different functions or associated parameters may subsequently be used to back-calculate the values when modelling real conditions (including the conditions of the original measurement!).

Databases should therefore be designed to hold sufficient information, so that little or no human intervention is required in the steps to modelling that follow, when most algebraic transformations of the data occur.

Second step: building datasets which are thermodynamically consistent

Storing as much data from the literature as possible introduces its own issue: the necessity then to produce a consistent set of mass balance equations from an inherently inconsistent body of primary and secondary data. Since achieving thermodynamic consistency between multiple reactions is combinatorial in nature, it can quickly become prohibitive in large multicomponent systems, i.e. attempting to check by hand all linear combinations arising in a real-size system exceeds human capability. As a consequence, thorough manual evaluations of thermodynamic data are confined to small chemical assemblages (e.g. [19]). To cope more generally, computational procedures that automatically achieve thermodynamic consistency are evidently required.

Testing the thermodynamic consistency of the data, either by manual or by computer automatic means, has the positive effect of detecting bad thermodynamic parameter 
values, transcription errors, etc. A further advantage of having a fully automatic approach is that keeping thermodynamic parameters up-to-date is simplified and the problem of the persistence of superseded values is avoided. Examples of the application of this approach by using JESS can be found in references [20,21] where antimony equilibria have been rationalised.

\section{The need to take into account the actual application of the calculation}

\section{Calculation-related considerations}

With most speciation programs having their own databases, the set of species whose concentrations or activities used in the calculation are predetermined and are the same for all calculations. However, in solving the mass balance equations, the optimum selection of species depends on which of them predominates at equilibrium; poor choices (i.e., when the concentrations of these species are too small) lead to difficulties due to mathematical ill-conditioning and failure of the calculation to converge. Therefore, the possibility of changing the basis/master species easily to solve a particular problem would be very useful. This requires that all the reactions can be reformulated in terms of the new basis species as and when required.

\section{Other sources of error and inaccuracy}

Aqueous solution chemistry underpins many predictive calculations in science and engineering including the evaluation of the environmental impact of toxic trace elements, the study of the anthropogenic contribution to global warming, assessing the security of new storage repositories in radioactive waste disposal and the improvement of industrial processes such as in hydrometallurgy. This means that chemical calculation needs to be applied to very different aqueous media, encompassing a wide range of ionic strengths, elemental composition and concentrations ranges. Different media will generally impose very different demands on the capabilities of chemical modelling, directly affecting the reliability of the results obtained. These factors need to be taken into account during any data assurance exercise. Two cases, coming from the direct experience of the authors, will be discussed here to illustrate this point.

\section{Applicability of existing equilibrium data to environmental systems}

When determining equilibrium constants, obviously only values for the complexes formed to a measurable extent in the experimental solutions can be determined; moreover, the accuracy and precision of the determined equilibrium constant values depend on the extent of formation of the corresponding species in the studied solutions. For this reason, a wide range of metal and ligand concentrations and metal-to-ligand ratios should be used to describe the system adequately. Even when this experimental approach is followed, however, the restrictive consequences of the limited analytical windows of each technique cannot be avoided. As is well known, every analytical technique is characterised by such an analytical window, i.e. a range of concentrations that give an analytical signal which can be adequately detected. For example, when glass electrode or ISE potentiometry are used in potentiometric determinations of equilibrium constants, metal and ligand concentrations should best be in the millimolar range. By definition, thermodynamic equilibrium constants applicable in such millimolar concentration solutions are valid at any concentration range. They can, and often are, used to simulate environmental conditions where trace elements are in the nanomolar or picomolar concentration range. However, what may happen is that the main species formed under these dilute conditions differ markedly from those at higher concentrations. This is the case particularly when polynuclear complexes predominate in solutions at millimolar concentrations. An important practical case of this occurs with thiol compounds, which have been suggested to complex trace elements in oceans [22-24], and with metal hydrolysis constants.

\section{Multicomponent concentrated aqueous electrolyte solutions}

Considerable difficulties persist in modelling the thermodynamics of multicomponent aqueous electrolyte solutions at high solute concentrations. This imposes restrictions on the applications of such models, which are needed in a wide variety of practical contexts such as in measurement science, oceanography, biochemistry and hydrometallurgy [25]. Although the Pitzer formalism [26] is frequently adopted in the chemical literature, it suffers from severe disadvantages. Especially when temperature and pressure changes are involved, the equations require many empirical parameters and are susceptible to correlation. They therefore tend to be ill-conditioned, and parameter fitting becomes highly dependent on the range of available data [27]. Their predictions are thus subject to serious error propagation. These issues become profoundly problematic with multicomponent systems because of the combinatorial increase in the number of Pitzer parameters needed and because of the relatively few experimental studies made on mixed electrolyte solutions. Reactions between the chemical species in solution, which often dominate in applied 
Fig. 1 Comparison of recommended and current methods for chemical thermodynamic modelling. $E C$ equilibrium constants, $L C$ linear combination

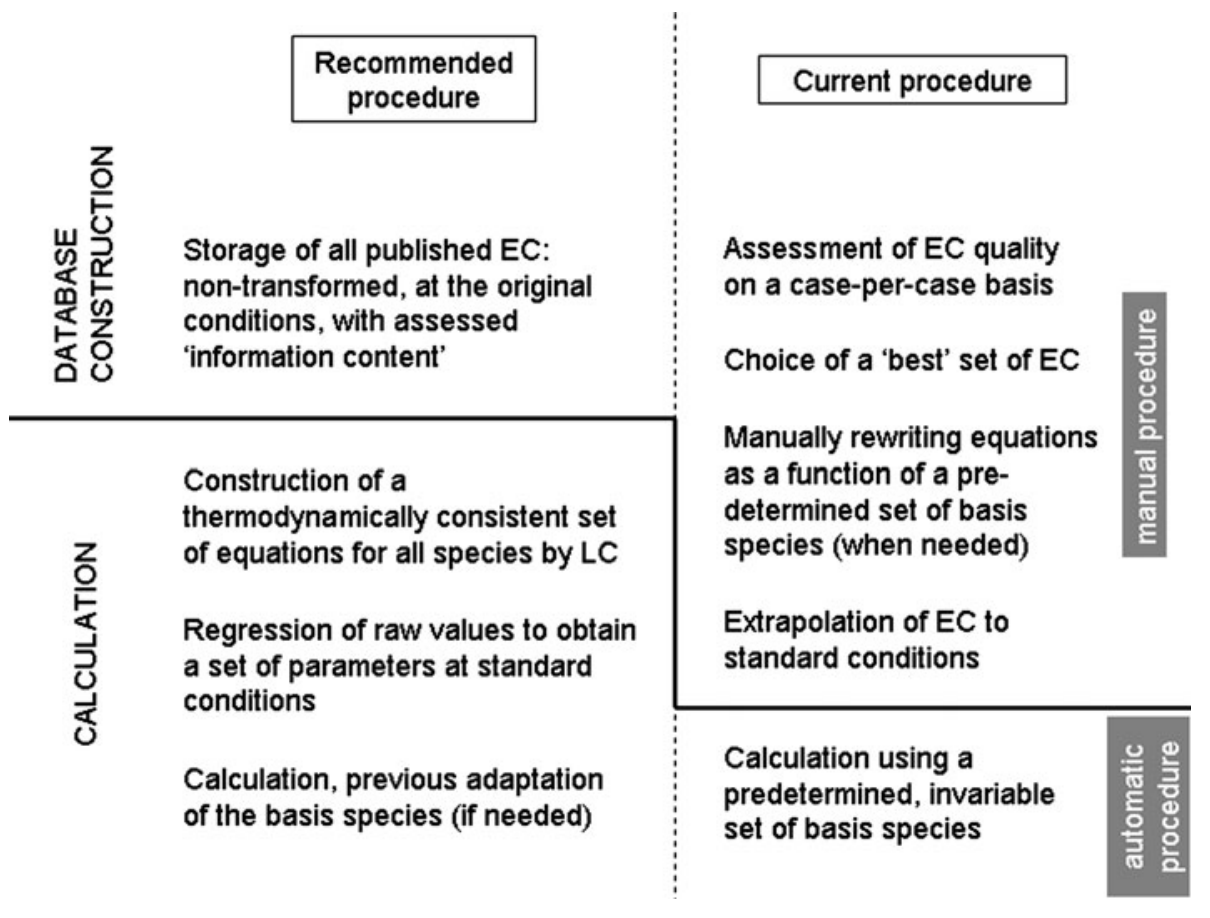

systems, add significant further complication. It has been evident for some time that a different, more fundamental, theoretical approach to modelling aquatic chemistry is needed to control the number of modelling parameters and to cope better with extrapolations into multicomponent spaces that have not been experimentally characterised.

\section{JESS}

A computer package (JESS, for Joint Expert Speciation System) has been developed by one of the authors (PM) along the lines described in this paper. JESS is a tool developed for modelling chemical speciation in complex aqueous environments, with a large integrated database of thermodynamic parameters for chemical reactions and an expert system capability for automatically achieving thermodynamic consistency. Details of JESS philosophy, structure and functioning have been published elsewhere [28-33].

\section{Conclusions}

The existence of serious discrepancies between published chemical thermodynamic data and the constraints this imposes when trying to use such data are well known. Accepted practices used to deal with the problem are based on gathering information, analysing its quality on a caseby-case basis, determining a selected set of best values and then using them to model the system. Although, thermodynamic consistency is achieved inherently by the predetermined set of basis species and selection of one formation parameter for each non-basis species, hidden discrepancies (e.g., information content which is being ignored or that may have even been overlooked or incorrectly eliminated by the database compiler) might remain. More importantly, since this manual approach involves a progressive building up of thermodynamic values from a foundation which fixes the results of each successive layer of work (see right-hand side in Fig. 1 for illustration), any change, correction or improvement cannot usually be made without starting again. For instance, this strategy does not readily allow for changes in the set of basis species to a particular problem, if required. In contrast, because most of the procedure is automated (Fig. 1), the approach advocated here has the ability of making changes easily (i.e., correction of errors, introduction of new constant values and change of ionic activity model.). This can then be combined with the optimal use of the information contained in all the existing available data, appropriately weighted according to their perceived quality.

\section{References}

1. Brecher K (1979) Albert Einstein: 14 March, 1879-18 April, 1955 A guide for the perplexed. Nature 278:215-218

2. OECD Chemical Thermodynamics Series, Update on the Chemical Thermodynamics of Uranium, Neptunium, Plutonium, Americium and Technetium, 2003, Volume 5

3. https://jess.murdoch.edu.au/, last accessed 20/04/2010 
4. Nancollas GH, Tomson MB (1982) Guidelines for the determination of stability constants. Pure Appl Chem 54:2675-2692

5. Braibanti A, Ostacoli G, Paoletti P, Pettit LD, Sammartano S (1987) Recommended procedure for testing the potentiometric apparatus and technique for the $\mathrm{pH}$-metric measurement of metalcomplex equilibrium constants. Pure Appl Chem 59:1721-1728

6. Tuck DG (1989) A proposal for the use of a standard format for the publication of stability constant measurements. Pure Appl Chem 61:1161-1163

7. Filella M, May PM (2005) Reflections on the calculation and publication of potentiometrically-determined formation constants. Talanta $65: 1221-1225$

8. www.nist.gov/srd/nist46.htm, last accessed 20/04/2010

9. www.oecdnea.org/html/dbtdb/info/publications/welcome.html, last accessed 20/04/2010

10. Portanova R, Lajunen LHJ, Tolazzi M, Piispanen J (2003) Critical evaluation of stability constants for $\alpha$-hydroxycarboxylic acid complexes with protons and metal ions and the accompanying enthalpy changes. Part II. Aliphatic 2-hydroxycarboxylic acids (IUPAC Technical Report). Pure Appl Chem 75:495-540

11. Powell KJ, Brown PL, Byrne RH, Gajda T, Hefter G, Sjöberg S, Wanner H (2005) Chemical speciation of environmentally significant heavy metals with inorganic ligands. Part 1: The $\mathrm{Hg}^{2+}$ $\mathrm{Cl}^{-}, \mathrm{OH}^{-}, \mathrm{CO}^{2-}, \mathrm{SO}^{2-}$, and $\mathrm{PO}^{3-}$ aqueous systems (IUPAC Technical Report). Pure Appl Chem 77:739-800

12. Anderegg G, Arnaud-Neu F, Delgado R, Felcman J, Popov K (2005) Critical evaluation of stability constants of metal complexes of complexones for biomedical and environmental applications (IUPAC Technical Report). Pure Appl Chem 77:1445-1495

13. Powell KJ, Brown PL, Byrne RH, Gajda T, Hefter G, Sjöberg S, Wanner H (2007) Chemical speciation of environmentally significant metals with inorganic ligands Part 2: The $\mathrm{Cu}^{2+}-\mathrm{OH}^{-}$, $\mathrm{Cl}^{-}, \mathrm{CO}_{3}{ }^{2-}, \mathrm{SO}_{4}{ }^{2-}$, and $\mathrm{PO}_{4}{ }^{3-}$ systems (IUPAC Technical Report). Pure Appl Chem 79:895-950

14. www.epa.gov/ceampubl/mmedia/minteq/, last accessed 20/04/ 2010

15. http://wwwbrr.cr.usgs.gov/projects/GWC_coupled/phreeqc/, last accessed 20/04/2010

16. Smith RM, Martell AE (1995) The selection of critical stability constants. In: Loeppert RA, Schwab AP, Goldberg S (eds) Chemical equilibrium and reaction models. SSSA special publication no. 42. SSSA, Madison WI, pp 7-29

17. Serkiz SM, Allison JD, Perdue EM, Allen HE, Brown DS (1996) Correcting errors in the thermodynamic database for the equilibrium speciation model MINTEQA2. Water Res 30:1930-1933

18. Onysko SJ, McNearny RL (1997) GIBBTEQ: A MINTEQA2 thermodynamic data error detection program. Groundwater 35:912-914
19. Königsberger E, Königsberger L-C, Gamsjäger H (1999) Lowtemperature thermodynamic model for the system $\mathrm{Na}_{2} \mathrm{CO}_{3}$ $\mathrm{MgCO}_{3}-\mathrm{CaCO}_{3}-\mathrm{H}_{2} \mathrm{O}$. Geochim Cosmochim Acta 63:3105-3119

20. Filella M, May PM (2003) Computer simulation of the low molecular-weight inorganic species distribution of antimony(III) and antimony(V) in natural waters. Geochim Cosmochim Acta 67:4013-4031

21. Filella M, May PM (2005) Critical appraisal of available thermodynamic data for the complexation of antimony(III) and antimony(V) by low molecular mass organic ligands. J Environ Monit 7:1226-1237

22. Le Gall AC, van den Berg CMG (1998) Folic acid and glutathione in the water column of the North East Atlantic. Deep-Sea Res I 45:1903-1918

23. Dupont CL, Moffett JW, Bidigare RR, Ahner BA (2006) Distributions of dissolved and particulate biogenic thiols in the subartic Pacific Ocean. Deep-Sea Res I 53:1961-1974

24. Chapman CS, Capodaglio G, Turetta C, van den Berg CMG (2009) Benthic fluxes of copper, complexing ligands and thiol compounds in shallow lagoon waters. Mar Environ Res 67:17-24

25. May PM (2006) Improved thermodynamic calculations for concentrated mixed electrolyte systems including ion pairing (or the absence of it). Mar Chem 99:62-69

26. Pitzer KS (1991) Ion interaction approach: theory and data correlation. In: Pitzer KS (ed) Activity coefficients in electrolyte solutions. CRC Press, Boca Raton, pp 75-153

27. Marshall SL, May PM, Hefter GT (1995) Least-squares analysis of osmotic coefficient data at $25{ }^{\circ} \mathrm{C}$ according to Pitzer's equation. 1. 1:1 electrolytes. J Chem Eng Data 40:1041-1052

28. May PM, Murray K (1991) JESS, a Joint Expert Speciation System-I. Raison d'être. Talanta 38:1409-1417

29. May PM, Murray K (1991) JESS, a Joint Expert Speciation System-II. The thermodynamic database. Talanta 38:1419-1426

30. May PM, Murray K (1993) JESS, a Joint Expert Speciation System-III. Surrogate functions. Talanta 40:819-825

31. May PM, Murray K (2001) Database of chemical reactions designed to achieve thermodynamic consistency automatically. J Chem Eng Data 46:1035-1040

32. May PM, Rowland D, Königsberger E, Hefter G (2010) JESS, a Joint Expert Speciation System-IV: A large database of aqueous solution physicochemical properties with an automatic means of achieving thermodynamic consistency. Talanta 81:142-148

33. Rowland D, May PM (2010) JESS, a Joint Expert Speciation System-V: Approaching thermodynamic property prediction for multicomponent concentrated aqueous electrolyte solutions. Talanta 81:149-155 